\title{
Functionalised microscale nanoband edge electrode (MNEE) arrays: the systematic quantitative study of hydrogels grown on nanoelectrode biosensor arrays for enhanced sensing in biological media $\dagger$
}

\author{
Andrew Piper, (D) a Ben M. Alston, ${ }^{\text {bc }}$ Dave J. Adams iD $\ddagger^{b}$ \\ and Andrew R. Mount (iD *a
}

Received 9th March 2018, Accepted 4th April 2018

DOI: $10.1039 / \mathrm{c} 8 \mathrm{fd} 00063 \mathrm{~h}$

\begin{abstract}
Nanoelectrodes and nanoelectrode arrays show enhanced diffusion and greater faradaic current densities and signal-to-noise ratios compared to macro and microelectrodes, which can lead to enhanced sensing and detection. One example is the microsquare nanoband edge electrode (MNEE) array system, readily formed through microfabrication and whose quantitative response has been established electroanalytically. Hydrogels have been shown to have applications in drug delivery, tissue engineering, and antibiofouling; some also have the ability to be grown electrochemically. Here, we combine these two emerging technologies to demonstrate the principles of a hydrogel-coated nanoelectrode array biosensor that is resistant to biofouling. We first electrochemically grow and analyze hydrogels on MNEE arrays. The structure of these gels is shown by imaging to be electrochemically controllable, reproducible and structurally hierarchical. This structure is determined by the MNEE array diffusion fields, consistent with the established hydrogel formation reaction, and varies in structural scale from nano (early time, near electrode growth) to micro (for isolated elements in the array) to macro (when there is array overlap) with distance from the electrode, forming a hydrogel mesh of increasing density on progression from solution to electrode. There is also increased hydrogel structural density observed at electrode corners, attributable to enhanced diffusion. The resulting hydrogel structure can be formed on (and is firmly anchored to/ through) an established clinically relevant biosensing layer without compromising detection. It is also shown to be capable, through proof-of-principle model protein studies using bovine serum albumin (BSA), of preventing protein biofouling whilst
\end{abstract}

${ }^{a}$ EaSTCHEM, School of Chemistry, The University of Edinburgh, Joseph Black Building, King's Building's, David Brewster Road, Edinburgh, EH9 3FJ, UK. E-mail: a.mount@ed.ac.uk

${ }^{b}$ Department of Chemistry, University of Liverpool, Crown Street, Liverpool, L69 7ZD, UK

${ }^{c}$ The Leverhulme Research Centre for Functional Materials Design, University of Liverpool, UK

$\dagger$ Electronic supplementary information (ESI) available. See DOI: 10.1039/c8fd00063h

‡ School of Chemistry, University of Glasgow, Glasgow, G12 8QQ, UK. 
enabling smaller molecules such as DNA to pass through the hydrogel matrix and be sensed. Together, this demonstrates a method for developing reproducible, quantitative electrochemical nanoelectrode biosensors able to sense selectively in real-world sample matrices through the tuning of their interfacial properties.

\section{Introduction}

Nanoelectrodes and hydrogels are each of growing interest in the field of biosensors. ${ }^{1-5}$ Hydrogels can be used to encapsulate and support biomolecules on a macroelectrode surface, thereby increasing the shelf life of these biosensor systems whilst not impeding their biomolecular function..$^{4-9}$ It has also been shown that hydrogels are permeable to small molecules ${ }^{10}$ and can be used to prevent electrode fouling in complex media such as human blood. ${ }^{11}$ Nanoelectrodes have been shown to possess enhanced mass transport which, coupled with a relatively small double layer charging current, yields a relatively large detection sensitivity and signal to noise ratio. ${ }^{\mathbf{1 2 , 1 3}}$

The use of nanoelectrode arrays containing multiple electrodes also allows the exploitation of the enhanced sensitivity of nanoelectrodes whilst measuring macroelectrode currents. ${ }^{14}$ We have detailed the reproducible fabrication through photolithographic processing of Microsquare Nanoband Edge Electrode (MNEE) arrays and shown through a combination of experiment and theory that steadystate and quantitative responses can readily and reproducibly be obtained, from which electroanalytical parameters can readily be extracted. ${ }^{\mathbf{1 2 , 1 3 , 1 5}}$ MNEE arrays are fabricated by the controlled geometrical etching of an array of square micron dimension cavities of controlled depth through a layer of Pt (of a defined nanometre thickness) sandwiched between two insulators of controlled thickness. This results in an array of micron scale cavities of defined separation, each of which contains a Pt nanoband running around the inside of the cavity at a controlled depth, defined by the etch depth and the thickness of the insulators. These electrode arrays are highly sensitive, and also highly reactive (consistent with the Pt nanoelectrode dimension). This is likely to result in enhanced biofouling in biosensing applications.

In this paper, the potential for combining these technologies through the functionalization of these nanoelectrode systems with hydrogels is therefore investigated, with a view to fabricating electrochemical biosensors which display both enhanced nanoelectrode array performance and the enhanced longevity and resistance to biofouling produced by hydrogels. To enable localized functionalization of the nanoelectrodes with hydrogels under spatial control, we have used the electrochemically controlled growth of low molecular weight hydrogels on electrodes. ${ }^{16} \mathrm{We}$ first establish the principle of electrochemical growth of hydrogels on MNEE arrays, then characterize the resulting hydrogel structures and finally determine the potential enhancement in biosensing characteristics of the combined system.

\section{Experimental}

\section{MNEE fabrication}

The MNEE array design, fabrication procedures and geometries are detailed elsewhere ${ }^{12,13}$ and shown below (Fig. 1). 

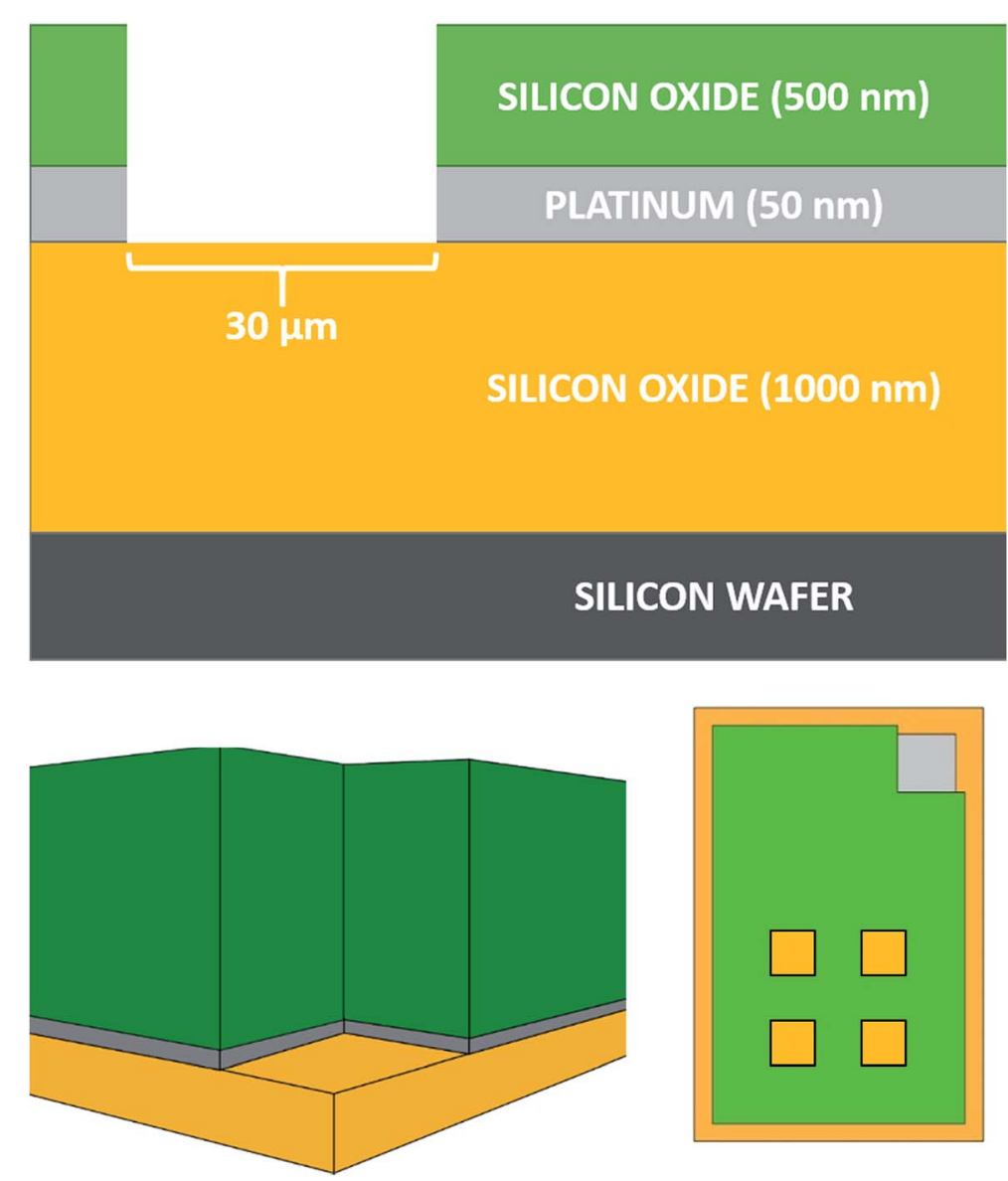

Fig. 1 Schematic diagrams (not to scale) of an MNEE array, displaying a schematic cross section of the device architecture (top), a cross section through one of the square cavities showing an MNEE (bottom left) and a schematic top view (bottom right, not to scale) showing the arrangement of 4 of the 1764 cavities and the bond pad connection in the top right hand corner.

All experiments were performed on these MNEE arrays, which consist of 1764 square cavities of $30 \mu \mathrm{m}$ edge length placed in a square arrangement, with each cavity separated by $90 \mu \mathrm{m}$ from its neighbors. The cavities were etched to a depth of $560 \mathrm{~nm}$, exposing a nanoband electrode of $50 \mathrm{~nm}$ thickness around the perimeter located at the base of each cavity. The total geometrical electrode surface area of the array is therefore $1.0584 \times 10^{-4} \mathrm{~cm}^{2}$.

\section{Electrochemical experiments}

All electrochemical measurements were made at a constant temperature of $25^{\circ} \mathrm{C}$, controlled by a water bath built in-house. They were performed in a Faraday cage using an Autolab PGSTAT128N with a FRA32 module (supplied by Metrohm Ltd.) controlled by a PC using NOVA 1.10 software. All potentials are reported with respect to the saturated calomel reference electrode (SCE, Accumet ${ }^{\circledR}$ 13-620-61, Fischer Scientific) unless otherwise stated, and the gauze counter electrode was 
made in-house using Pt gauze and wire (Sigma-Aldrich). All solutions were prepared using deionized water (18.2 $\mathrm{M} \Omega \mathrm{cm}^{-1}$, Millipore MilliQ) with sodium chloride (BioXtra $\geq 99.5 \%$ ), potassium ferricyanide (ACS reagent $\geq 99 \%$ ), potassium ferrocyanide (ACS reagent $\geq 99 \%$ ) and hydroquinone (reagentplus ${ }^{\circledR}$ $\geq 99.5 \%$ ) all supplied by Sigma Aldrich. All pH measurements were performed using a Fisherbrand Hydrus $400 \mathrm{pH}$ meter with a Mettler Toledo InLab®Routine Pro pH probe. Electrochemical impedance spectroscopy (EIS) measurements were carried out in an aqueous solution of potassium ferrocyanide $(1 \mathrm{mM}$, Sigma Aldrich) and potassium ferricyanide (1 mM, Sigma Aldrich) in KCl (0.1 M, Sigma Aldrich) background electrolyte. EIS was performed by applying a sinusoidal potential of $10 \mathrm{mV}$ rms superimposed on the open circuit potential (OCP), to ensure no overall faradaic reaction. 50 logarithmically spaced frequencies were applied in the range between $100 \mathrm{kHz}$ and $0.1 \mathrm{~Hz}$. The OCP was measured before each EIS measurement and, as expected for the ferricyanide/ferrocyanide couple was typically between +165 and $+170 \mathrm{mV}$ with respect to SCE.

\section{Carbazole-alanine synthesis and hydrogel formation}

The carbazole-alanine hydrogel was prepared as described previously. ${ }^{16}$ A solution of the gelator was prepared by stirring carbazole-alanine $\left(2 \mathrm{mg} \mathrm{mL}^{-1}\right)$ in aqueous sodium hydroxide $(6.75 \mathrm{mM})$ at room temperature overnight until it had fully dissolved. Aqueous $\mathrm{HCl}(1 \mathrm{M})$ and $\mathrm{NaOH}(6.75 \mathrm{mM})$ solutions were used as appropriate to adjust the $\mathrm{pH}$ to 8 . Hydroquinone $\left(2.88 \mathrm{mg} \mathrm{mL} \mathrm{m}^{-1}\right.$, Sigma Aldrich) and $\mathrm{NaCl}$ (0.1 M, Sigma Aldrich) were added as the $\mathrm{pH}$ swing inducer and the background electrolyte respectively. Gelation was induced using chronopotentiometry, holding the MNEE array working electrode at $+100 \mu \mathrm{A}$ unless otherwise stated in a threeelectrode system using a Pt wire as a pseudo-reference electrode.

\section{Imaging (SEM and confocal microscopy with Nile blue stain)}

SEM images were obtained using a Hitachi S-4800 FE-SEM at $3 \mathrm{keV}$. Hydrogels were electrochemically grown on to the MNEE and fixed onto aluminium SEM stubs with carbon tabs and air dried for direct imaging. The MNEE arrays were gold coated for 1.5 minutes at $20 \mathrm{~mA}$ using a EMITECH K550X sputter coater prior to imaging.

Confocal microscopy images were obtained on a Zeiss LSM510 and a Plan-Apo $100 \times(1.4 \mathrm{NA}$ oil $)$ and $63 \times(1.46 \mathrm{NA}$ oil $)$ objective. The gelator solution was prepared as described previously, with the addition of $0.001 \mathrm{M}$ Nile blue. Nile blue was excited at $633 \mathrm{~nm}$ using a helium neon laser and its emission was detected between 638 and $758 \mathrm{~nm}$ using a pinhole diameter of 1 Airy unit (approx. $0.8 \mu \mathrm{m}$ resolution). The working electrode was placed in an uncoated borosilicate MatTek dish with a $20 \mathrm{~mm}$ diameter glass coverslip. Images were analyzed using the Zeiss AIM software (Zeiss, Jena, Germany) and ImageJ software.

\section{PNA SAM formation, DNA target binding and biofouling studies}

PNA SAM formation was carried out using 1.5 nM PNA (Panagene, Daejeon, South Korea), $150 \mathrm{nM}$ tris-2-carboxymethylphosphine (TCEP, Sigma-Aldrich) and $30 \mathrm{nM}$ 6-mercaptohexan-1-ol (MCH, Sigma-Aldrich) in a $50: 50$ mixture by volume of DMSO and deionized water. $50 \mu \mathrm{L}$ of this mixture was placed on the MNEE array for 1 hour to form the PNA SAM on the electrode surfaces, followed by growth of the carbazole-alanine hydrogel galvanostatically in gelator solution at $+100 \mu \mathrm{A}$ for 
$10 \mathrm{~s}$. The functionalized MNEE array was then exposed to a $50 \mu \mathrm{L}$ droplet of 100 $\mu \mathrm{M}$ aqueous solution of 30-mer complementary target DNA (Sigma-Aldrich) sequence:

5'-GTATGCTTTGGTCTTTCTGCATTCCTGGAA-3' for 30 minutes. Measurement of target binding involved performing EIS as above, using redox buffer $(1 \mathrm{mM} /$ $1 \mathrm{mM}\left[\mathrm{Fe}(\mathrm{CN})_{6}\right]^{3-} /\left[\mathrm{Fe}(\mathrm{CN})_{6}\right]^{4-}$, in aqueous $1 \times$ Tris- $\left.\mathrm{HCl} \mathrm{pH} 4.3\right)$ so as to ensure gel stability. For biofouling kinetic experiments, this redox buffer was also used, as it has been previously established that bovine serum albumin (BSA) solution is stable at this $\mathrm{pH} .{ }^{17}$ BSA (Sigma Aldrich, UK) was purchased as a lyophilized powder and made up to a final $100 \mu \mathrm{M}$ concentration, followed by $\mathrm{pH}$ readjustment as required.

\section{Results and discussion}

The carbazole-alanine forms hydrogels by self-assembling into fibrous structures, induced by a $\mathrm{pH}$ decrease. In previous work, the electrochemical oxidation of hydroquinone at a macroelectrode surface has been used to lower the $\mathrm{pH}$ locally, so as to grow a hydrogel matrix. ${ }^{16}$ The linear diffusion regime established across these electrodes is assumed to produce a relatively homogeneous hydrogel. MNEE arrays have established enhanced steady-state mass transport with a mixture of coupled diffusional regimes (highly enhanced nanoscale diffusion near band electrodes, enhanced microscale diffusion within and immediately outside each cavity and macroscale diffusion where cavity array diffusional fields overlap). ${ }^{13}$ This was thought likely to lead to spatially inhomogeneous $\mathrm{pH}$ decrease and hydrogel formation, so the appropriate electrochemical hydrogel growth conditions and resulting hydrogel structures first needed to be established for MNEE arrays.

Following the previous electrochemical hydrogel procedure, ${ }^{16}$ Fig. 2 a shows a typical potential time transient for hydrogel formation at the MNEE array, along with an image of the resulting hydrogel (Fig. 2b). During the gelation process, following the initial establishment of the characteristic hydroquinone oxidation potential above $+1.0 \mathrm{~V}$ reported previously, over short periods of time $(t<400 \mathrm{~s})$ the potential was seen to increase essentially linearly to around $+1.3 \mathrm{~V}$, consistent with the increased overpotential required to deliver this oxidation current through the growing hydrogel. After $400 \mathrm{~s}$, the potential was seen to rise relatively rapidly to over $+2.0 \mathrm{~V}$, consistent with mass transfer of hydroquinone to the electrode surface being insufficient to support this current, and the growing importance of solvent oxidation and oxygen gas formation. This gas formation is the most likely cause of the bubbles that can be observed in the hydrogel in Fig. 2b. As these oxygen bubbles will compromise the hydrogel structure, growth was limited to times less than $400 \mathrm{~s}$, where significant amounts of bubbles do not form.

To investigate the different stages of electrochemical hydrogel formation, galvanostatic growth using this procedure was carried out for successive 5-second periods, followed by optical (using scanning electron microscopic, confocal microscopic and light microscopic imaging) and electrochemical characterization.

Fig. 3 shows typical Nyquist plots after these successive growth periods.

It is reassuring that the measured impedance values increased with longer hydrogel formation times, consistent with a progressive increase in surface hydrogel formation. The fits to these EIS data enable quantitative assessment of 
A
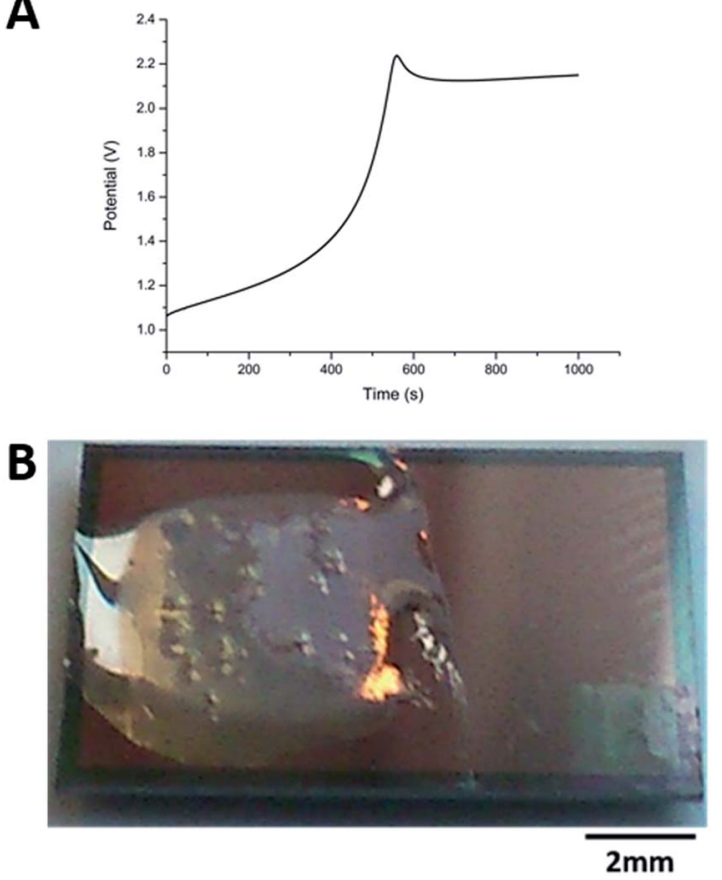

Fig. 2 (A) Typical potential-time transient recorded during $15 \mathrm{~min}$ of galvanostatic hydrogel growth at $+100 \mu \mathrm{A}$ on an MNEE array and (B) a photograph of the resulting hydrogel. Note that potentials reported are with respect to the platinum pseudoreference.

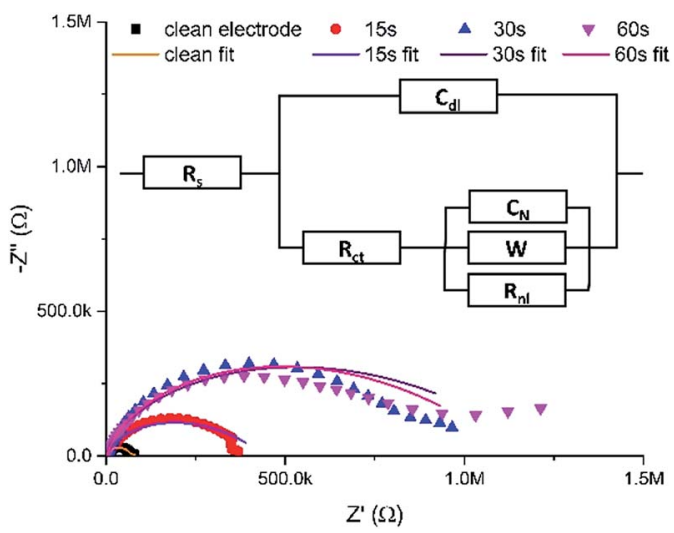

Fig. 3 Typical EIS Nyquist data (points) for a clean MNEE array and an MNEE array after a total of 15 seconds, 30 seconds and 60 seconds of hydrogel growth, along with fits of these data (lines) to the general equivalent circuit (inset). Note that Table S1 in the ESI $\dagger$ contains the values for the fits of these data to the appropriate limiting case for the general equivalent circuit (inset). Also note that this is an extension of the established MNEE equivalent circuit, ${ }^{13}$ with an additional Warburg element to account for within hydrogel diffusion. All fits give relatively low fit values (with $\chi^{2}<0.156$ ). 
hydrogel characteristics (vide infra). The light microscopy images (Fig. 4) also confirm the progressive growth of hydrogel.

The magnitude of the galvanostatic current and the potential profile suggest that the hydrogel growth is largely determined by diffusion, with hydroquinone diffusion and reaction at the electrode producing the required local $\mathrm{pH}$ decrease for hydrogel formation within the diffusion layer through proton production. ${ }^{\mathbf{1 6}}$ Under these conditions, the thickness of the diffusion layer, $l$, as a function of time, $t$, can be estimated by:

$$
t \approx l^{2} / D
$$

where $D$ is the diffusion coefficient. This is an estimate, as it is not firmly established whether the gel growth is limited by the flux of hydroquinone to the electrode rather than the local $\mathrm{pH}$ (controlled by the flux of $\mathrm{H}_{3} \mathrm{O}^{+}$away from the electrode). The value of $D$ is also likely to decrease within the hydrogel.

\section{Characterization of hydrogel growth; spatial determination of the hydrogel structure}

$\boldsymbol{t}=\mathbf{5}$ s. Taking literature values of $D$, the diffusion coefficient for hydroquinone, as $2.7 \times 10^{-6} \mathrm{~cm}^{2} \mathrm{~s}^{-1},{ }^{18}$ and for $\mathrm{H}^{+}$as $4.5 \times 10^{-5} \mathrm{~cm}^{2} \mathrm{~s}^{-1},{ }^{19}$ after 5 seconds the characteristic diffusion layer thickness for hydroquinone would be of the order of $40 \mu \mathrm{m}$; for protons it would $225 \mu \mathrm{m}$. Given the likely decrease in $D$ and the
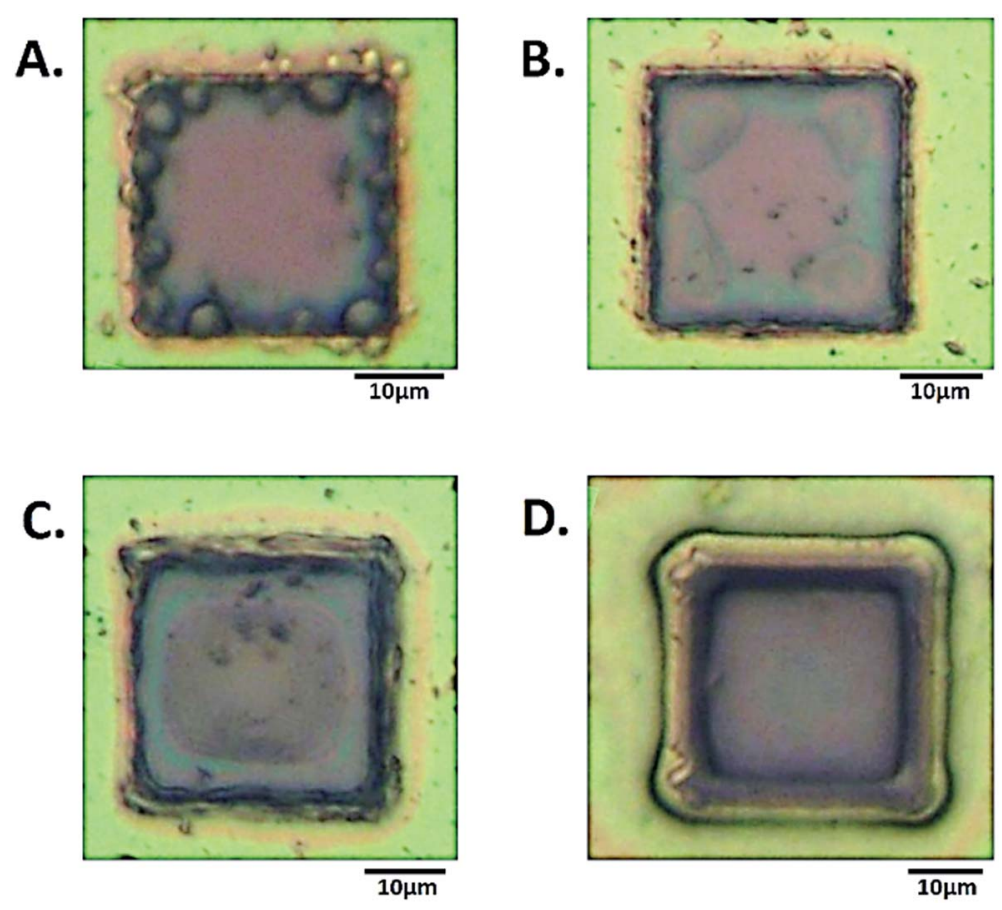

Fig. 4 Light microscopy images of a single square in the MNEE array after (A) 5 seconds, (B) 10 seconds, (C) 15 seconds, (D) 20 seconds growth at $50 \mu \mathrm{A}$. 
fact that the $\mathrm{pH}$ excursion is likely to be lower, due to buffering by dissolved carbonate species, ${ }^{19,20}$ and the dilution caused by the enhanced proton nanoscale diffusion, it is satisfying that the corresponding light microscopy image (Fig. 4a) indicates hydrogel growth to a comparable distance of around ten microns.

It is also satisfying that the EIS fit values of $R_{\mathrm{s}}, R_{\mathrm{ct}}$ and $C_{\mathrm{dl}}$ remain relatively constant at $164 \pm 5 \Omega, 19 \pm 6 \mathrm{nF}$ (corresponding to $180 \pm 60 \mu \mathrm{F} \mathrm{cm}^{-2}$ a typical double layer capacitance for an uncoated electrode of these dimensions ${ }^{21}$ ) and 19 $\pm 3 \mathrm{k} \Omega$ during hydrogel growth, (Fig. S1 and Table S1 in the ESI $\dagger$ ). This confirms that the hydrogel is an open structure which does not hinder significantly the passage of electrolyte ions, and that the electrochemical response at the underlying electrode remains unchanged. However, there is a significant increase in $R_{\mathrm{nl}}$ and a decrease in $Y_{0}$ (obtained from $W$ ) compared to the clean MNEE array after 5 seconds of hydrogel growth, with the observed mean value of $D$ for ferri/ ferrocyanide dropping from the accepted literature value ${ }^{22}$ of $(7.7 \pm 0.03) \times$ $10^{-6} \mathrm{~cm}^{2} \mathrm{~s}^{-1}$ to $(0.89 \pm 0.01) \times 10^{-6} \mathrm{~cm}^{2} \mathrm{~s}^{-1}$ from $R_{\mathrm{nl}}$ and $(0.26 \pm 0.02) \times 10^{-6}$ $\mathrm{cm}^{2} \mathrm{~s}^{-1}$ from $Y_{0}$ (see eqn $\mathrm{S} 1$ and $\mathrm{S} 2 \dagger$ ). This indicates that the progressive growth of the gel matrix does cause a decrease in $D$. It is interesting that the value derived from $Y_{0}$ (due to linear diffusion close to the electrode) is now lower than that derived from $R_{\mathrm{nl}}$ hemispherical diffusion across the diffusion layer formed at each cavity, which suggests the inhibition of mass transport due to a relatively dense hydrogel formed at the electrode surface. This is to be expected, as electrochemical hydrogel formation must be localized at the nanoelectrode, where the hydrogel must therefore be of nanodimensions, producing a nanointerface. This is confirmed by confocal microscopy (Fig. 5a) and SEM (Fig. 6a), which show that hydrogel is firmly anchored to, and grows from the entire nanoband.

$\boldsymbol{t}=\mathbf{1 0} \mathrm{s}$. The confocal microscopy (Fig. 5b) indicates a clear growth in the area of enhanced dye emission, consistent with the expected progressive growth of the hydrogel. It is also interesting that there are areas of markedly enhanced dye emission, emanating from the corners. This indicates increased gelator density, and is consistent with the previously predicted and observed enhanced currents for microsquare versus microdisc electrodes, due to enhanced square corner diffusion and reaction. ${ }^{13,15}$ It is interesting that SEM images taken at $t=5 \mathrm{~s}$ also indicate such enhanced xerogel corner density (Fig. S2 $\dagger$ ). Notwithstanding the expected conversion of hydrogel to collapsed gelator on the electrode surface due to vacuum desiccation, the SEM image (Fig. 6a and b and close ups in Fig. S2 $\dagger$ ) confirms this increase in gelator at the corners.

$\boldsymbol{t}=\mathbf{2 0}$ s. After 20 seconds, both the light microscopy and SEM images (Fig. 6c and d) indicate that hydrogel has grown sufficiently to fill each cavity in the array. This is consistent with the confocal microscopy image (Fig. 5c), which is a circular 2D slice of the near hemispherical 3D hydrogel. This image indicates the continuation of spatial heterogeneity in the growing hydrogel structure, built on the observed structure in Fig. 5b and consistent with the effects of diffusion to the cavity. This hemispherical hydrogel is consistent with the previous postulation of the origin of $C_{\mathrm{N}}$ in the MNEE equivalent circuit as being due to a relatively rapid reaction of the essentially hemispherical volume above the cavity, brought about by enhanced nanoscale diffusion induced by the nanoband electrode around the cavity edge. ${ }^{13}$

$\boldsymbol{t}=20-30 \mathrm{~s}$ and beyond; hemispherical growth to array overlap. On a timescale of 20-30 seconds, the diffusion fields (and therefore hemispherical hydrogel formation fields) are expected to continue to grow out from each cavity. This is 

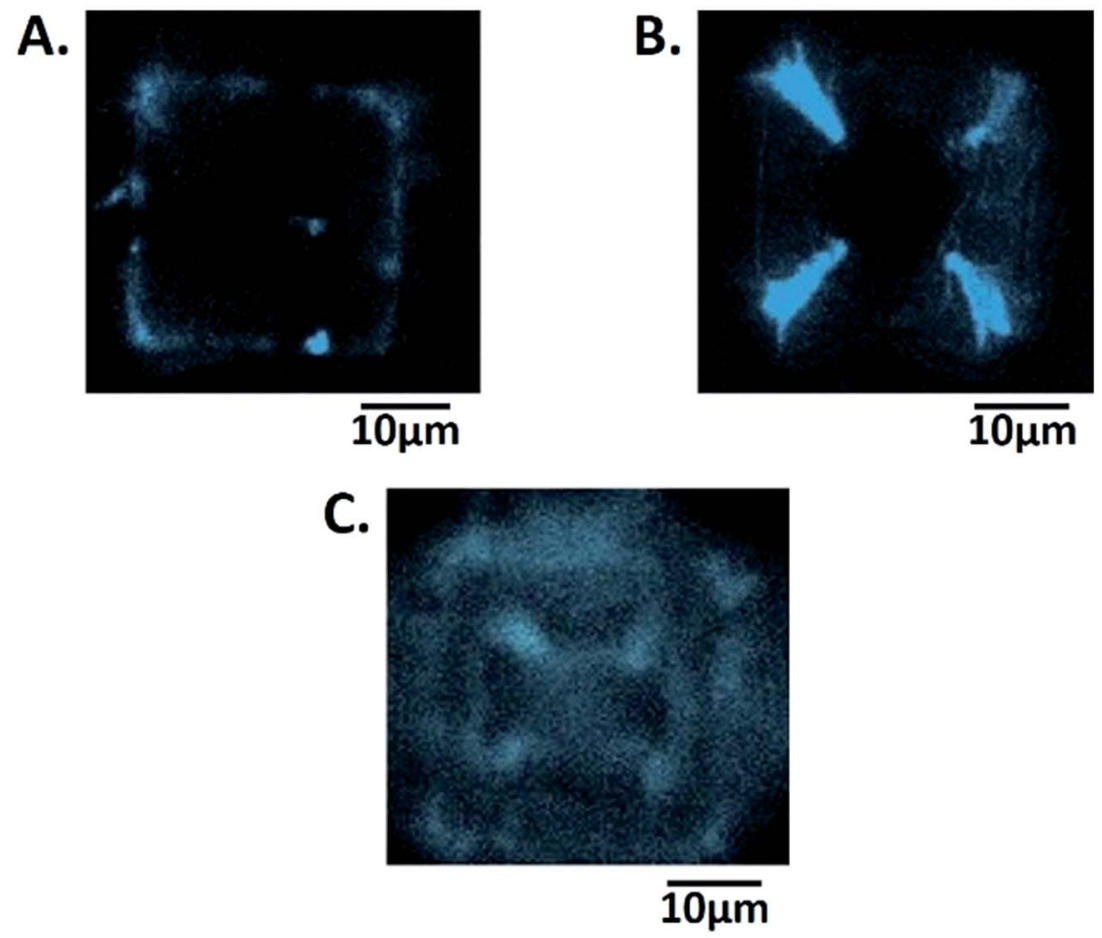

Fig. 5 Confocal microscopy images of a single square cavity in the hydrated MNEE array after (A) 5 seconds (B) 10 seconds (C) 20 seconds of hydrogel growth, following staining and imaging with Nile blue.

shown by the light microscopy image from a dehydrated hydrogel grown for 30 seconds (Fig. 7a), which shows some elements of the growing hydrogel have fused with neighbours, such that their dehydrated, collapsed structure retains the evidence of this fusion.

Further prolonged hydrogel growth leads to progressive fusion of all neighbouring hydrogel elements, and the establishment of a hydrogel structure which covers the entire electrode area. This is consistent with the expected elemental diffusion field overlap and the establishment of planar diffusion across the array. Light microscopy of the hydrated hydrogel (Fig. 6b) indicates this hydrogel has a relatively open micron-scale structure in comparison to the denser, hemispherical structure imaged at and around each cavity due to the earlier stages of hydrogel formation.

These structural data provide a clear rationale for each of the limiting cases of the global equivalent circuit used to analyse the impedance data (Fig. 2). Initially, prior to hydrogel formation, as expected the data fits the established clean MNEE array circuit, ${ }^{8}$ with no Warburg impedance $\left(Y_{0} \rightarrow \infty\right)$. After initial hydrogel growth, there is a growing contribution from $W$, which we attribute to the diffusion of redox species within the hydrogel. Finally, on array overlap and continued hydrogel film growth across the entire MNEE array, a further semicircular feature is observed which we attribute to an additional series circuit element of a capacitor and resistor in parallel due to charge transfer into this film (Fig. S1 and Table 
A

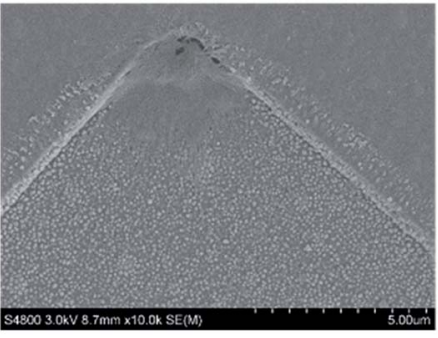

C

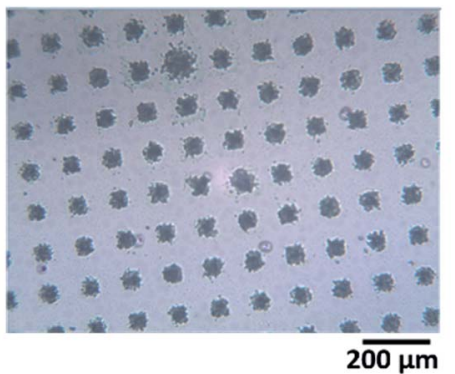

B

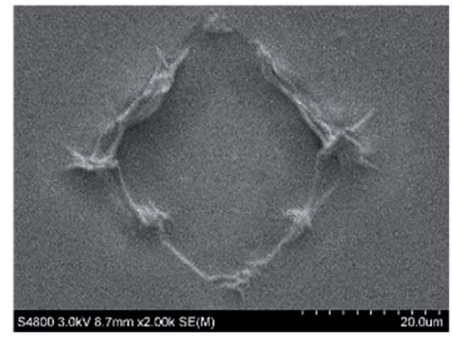

D

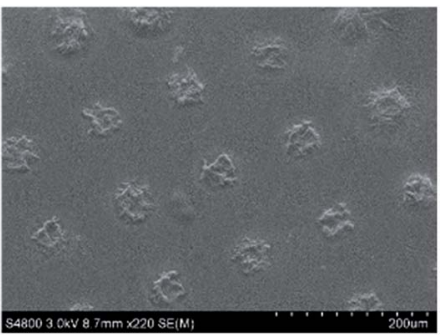

Fig. 6 Scanning electron microscopy (SEM) images of the MNEE array after (A) 5 seconds and (B) 10 seconds growth at $50 \mu \mathrm{A}$. A close up of the electrode in (A) can be found in Fig. S3† showing that gel is growing across the entire electrode but enhanced growth is clearly occurring in the corners. (C) and (D) are light microscopy and SEM images of a collapsed (dehydrated) hydrogel, respectively, after 20 s, which show that hydrogel has completely filled each cavity in the array.

$\mathrm{S} 1 \dagger)$. It is satisfying that throughout these different stages of growth the values for $R_{\mathrm{s}}, R_{\mathrm{ct}}$ and $C_{\mathrm{dl}}$ remain constant, and $R_{\mathrm{nl}}$ also remains essentially unchanged. This is consistent with the formation of a spatially heterogeneous hydrogel, whose local structure is determined by the local diffusional characteristics at hydrogel formation and then retained.

\section{Global control of the hydrogel structure}

In addition to the controlled spatial hydrogel heterogeneity induced by the MNEE diffusion fields, Fig. 7 shows that the current at which the gel is grown also
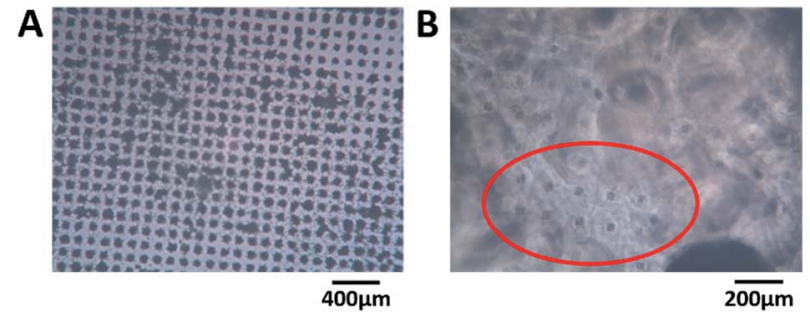

Fig. 7 Light microscope images of (A) dehydrated hydrogel after $30 \mathrm{~s}$ growth and (B) hydrated hydrogel after 15 min growth. Note that after 30 seconds there is the start of neighboring hydrogel coalescence. The gel has obscured the majority of the array but thinner patches of gel exist where array elements, and the hemispherical shaped hydrogel surrounding them, are observable. Note the black spot in the bottom right is due to a void in the hydrogel arising from growth around a gas bubble (for similar voids, see Fig. 2). 
determines the density of the organic scaffold and hence the porosity of the gel formed. Fig. 8 shows a comparison of the EIS spectra of hydrogels grown for 15 seconds at 50 and $100 \mu \mathrm{A}$ respectively (which should give similar diffusion fields and hydrogel volumes due to the fixed time). It is clear that the impedance values for the reaction and diffusion of ferri/ferrocyanide through the hydrogels formed at the lower current are markedly higher, and that the fit parameters (Table S1 $\dagger$ ) show a statistically significant and marked decrease in $Y_{0}$ and a corresponding increase in $R_{\mathrm{nl}}$, attributable to a decrease in the mean diffusion coefficient $D$ of the order of a factor of 4 for ferri/ferrocyanide (from around $2 \times 10^{-7} \mathrm{~cm}^{2} \mathrm{~s}^{-1}$ at $100 \mu \mathrm{A}$ to between $4-8 \times 10^{-8} \mathrm{~cm}^{2} \mathrm{~s}^{-1}$ at $50 \mu \mathrm{A}$ ), indicating the formation of a denser, less porous hydrogel compared to that formed at the higher current. There is also a small decrease in $C_{\mathrm{dl}}$, which would be consistent with an increase in the density of the organic scaffold of the nanodimensional hydrogel at the electrode surface. Such an increase in density at lower current would be consistent with an increase in the local concentration of gelator species due to a reduction in the importance of mass transfer.

\section{Feasibility of biosensing through a hydrogel on a MNEE array}

The ability to vary the overall density and hence porosity of the gel, combined with the established controlled and reproducible spatial variation in hydrogel structure, could be of value in the tuning of molecular filtration properties of these hydrogels. One potential application would be the production of a surface layer which inhibits or prevents electrode sensor biofouling. ${ }^{11}$ Biofouling, especially of the nanointerface of nanoelectrodes, is an area of great interest as moves are made towards implantable diagnostic devices. ${ }^{23,24}$ All point-of-care diagnostics would benefit from being able to detect analytes in complex media without the need for pre-treatment, which can be expensive and time consuming., ${ }^{4,23}$ One potential use of electrochemically produced hydrogels would be if they could be grown over established electrochemical sensing/detection systems, thereby conferring surface anti-biofouling, without compromising target detection. The feasibility of this approach was therefore tested, by using the model system of an

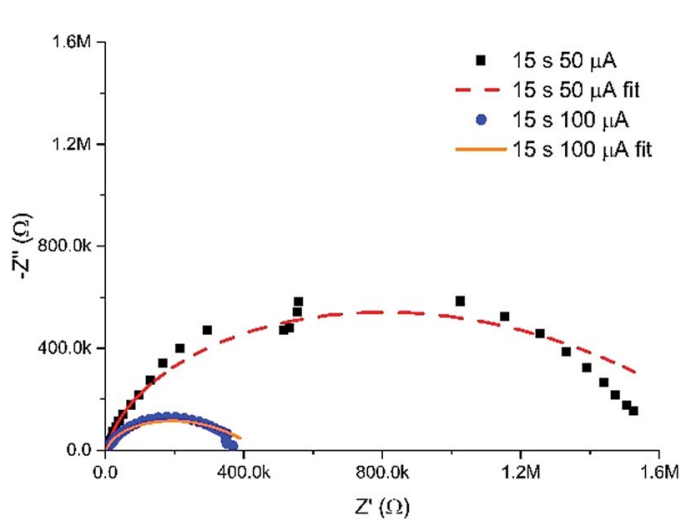

Fig. 8 Nyquist plots in redox buffer of hydrogels grown for 15 seconds at 50 and $100 \mu \mathrm{A}$, along with their associated fits to the equivalent circuit (with fit parameters given in Table $\mathrm{S} 1 \dagger$ ). 
established biomolecular sensing film readily formed through creating a selfassembled monolayer on an electrode. ${ }^{25}$ The previously established and clinically relevant DNA sensing protocol for the detection of the MRSA mecA cassette was chosen to determine the ability of the electrode to retain its function as a working biosensor following electrochemical hydrogel formation. The protocol involved forming a self-assembled monolayer (SAM) using a peptide nucleic acid (PNA) probe with a sequence homologous to the MRSA mecA cassette. A spacer molecule (6-mercaptohexan-1-ol, $\mathrm{MCH}$ ) was also present in the solution (and film) in a $15: 1$ ratio with the PNA to optimize surface coverage. ${ }^{25}$ The carbazolealanine hydrogel matrix was then grown through and over this film for 10 seconds at $50 \mu \mathrm{A}$ as above before being incubated with a 15-mer target DNA $(100 \mu \mathrm{M})$ for 30 minutes. The electrode was then placed in the redox buffer and hybridization measured as above using EIS. This sensing protocol has previously been shown to not exhibit non-specific binding to mismatched DNA target sequences. ${ }^{25}$

Fig. 9a shows the Nyquist plots obtained using this protocol, which show the expected increases in the impedance, first on going from a clean MNEE nanoelectrode array to an array with a blocking PNA SAM probe film, then with an additional layer of hydrogel electrochemically grown on the surface, and finally, when target passed through the hydrogel and bound to the PNA probe. Detailed analysis revealed the expected increase in $R_{\mathrm{ct}}$ when going from a clean electrode to one that has a SAM film on the surface, whilst $R_{\mathrm{nl}}$ stayed constant, which confirms that there was no effect on $D$ (mass transfer to the electrode). Growing the hydrogel over the SAM was then seen to increase $R_{\mathrm{n} 1}$ significantly, as expected, such that the two near semicircular features are no longer distinguishable by eye. This confirms the growth of the hydrogel matrix, within which there is the corresponding expected decrease in diffusional rate. It is interesting that is also a significant increase in $R_{\mathrm{ct}}$, since it has been established that this charge transfer response is largely determined by redox reaction through pinhole defects in the SAM layer formed on multicrystalline electrode surfaces. ${ }^{26-28}$ This suggests that the local enhanced $\mathrm{pH}$ change and hydrogel growth increases the observed charge transfer resistance through constricting the size, and/or blocking at least some, of these pinholes as a result of the creation of hydrogel anchor points to the underlying electrode, which is consistent with the previously observed adherence of the hydrogel. The final sensing step again resulted in the expected significant increase in $R_{\text {ct }}$ (without a change in $R_{\mathrm{nl}}$ ), due to the formation of the more blocking PNA-DNA duplex SAM film at the electrode surface. It is satisfactory that this relative increase in $R_{\mathrm{ct}}$ on target binding is comparable to that observed previously for this biosensor system, ${ }^{25}$ which confirms that the biosensing function is not significantly affected, even after the growth of the surface hydrogel.

To test the ability of this carbazole-alanine hydrogel to prevent biofouling, a clean MNEE was placed in redox buffer containing $1 \mu \mathrm{M}$ bovine serum albumin (BSA). It has previously been reported that BSA is a $\sim 66.5 \mathrm{kDa}$ protein, stable in its wildtype form at the redox buffer $\mathrm{pH}$ of 4.3 , that has been shown to readily foul a clean electrode surface. ${ }^{17,29}$ Fig. $8 \mathrm{~b}$ shows the resulting values of $R_{\mathrm{ct}}$ obtained from successive EIS measurements as a function of time. These demonstrate the expected and progressive significant increase in $R_{\mathrm{ct}}$ due to surface nanoelectrode biofouling. In contrast, after surface hydrogel growth at $100 \mu \mathrm{A}$ for $20 \mathrm{~s}$ the values of $R_{\mathrm{ct}}$ were seen to remain low and invariant with time. This stark contrast between the two signals clearly demonstrates the ability of the hydrogel, with its 

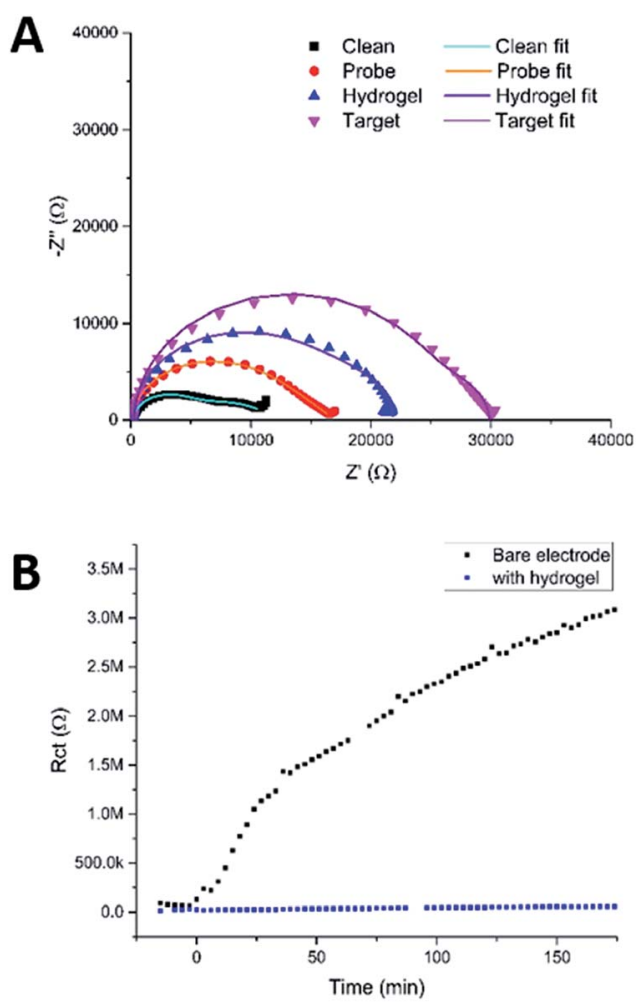

Fig. 9 (A) Nyquist plots of a clean MNEE array, an array with a PNA probe SAM layer, with a hydrogel grown over and around the SAM film and after binding of target DNA that has penetrated the hydrogel and bound to the probe. Circuit fits can be found in Table S2. $\uparrow$ (B) Fitted values of $R_{\mathrm{ct}}$ obtained by taking consecutive measurements of an electrode in redox buffer containing $100 \mu \mathrm{M}$ BSA with and without hydrogel on the electrode surface. The points below 0 min were repeat measurements taken on a clean electrode, prior to exposure to BSA, as a control.

increasingly dense structure from bulk solution to electrode, to prevent BSA biofouling of the underlying nanoelectrode array.

\section{Conclusions}

This work has established that spatially structured hydrogels can be electrochemically grown reproducibly and controllably on the surface of MNEE arrays, and that this growth does not compromise the sensing activity of a biosensing SAM nanointerfacial layer. It also demonstrates that the hydrogel can be used to prevent the non-specific binding of the biologically relevant $66 \mathrm{kDa}$ BSA protein. These are the essential proofs of principle required to demonstrate the feasibility of electrochemical target detection from complex media, which contain biologically relevant proteins, by using SAM probe-target binding at nanoelectrodes capped with hydrogel. Further work required to develop this system includes the exploitation of the tuning of gelation and the resulting hydrogel structure (as demonstrated by control of the hydrogel current) to enable optimization of protein-target selectivity for the variety of proteins found in such media. 
It is also interesting that experiments attempting to measure target DNA binding through the hydrogel in a solution containing BSA produced no measurable change in $R_{\mathrm{ct}}$. This can be explained by the non-specific binding of the BSA to these target DNA oligonucleotides; whilst Barton et al. have shown that BSA does not bind to DNA at biologically relevant neutral $\mathrm{pHs}^{30}{ }^{30}$ these experiments were run at $\mathrm{pH} 4.3$ so as to not destabilise the hydrogel structure. As the isoelectric point of BSA is $\mathrm{pH} 4.7,{ }^{31}$ under these conditions the positively charged BSA is likely to bind electrostatically to negatively charged DNA target in the system, thereby preventing its detection. Thus, although this hydrogel is likely to be able to enable target biosensing in acidic biological media, such as the stomach, this highlights the desirability of developing hydrogels that can be both electrochemically manufactured and stable at neutral $\mathrm{pH}$ in order to enable the detection of analytes in a wider range of biological media.

Finally, to address the added value of these nanoelectrodes and their resulting functionalisation to form nanointerfaces, it is worth considering the degree and homogeneity of spatial control of hydrogel formation that could also be achieved when employing established micron scale electrode systems such as a microdisc or microsquare electrode. Although both of their overall steady-state, mass transport limited electrochemical responses can be categorised essentially in terms of hemispherical diffusion to the electrode surface, non-uniform fluxes are seen across these electrode surfaces with markedly enhanced solution mass transfer rates to the electrode edge regions of both (and, like the corners of square nanocavity, Fig. 5b, particularly to the corners of the microsquare) when compared to the rest (the inner surface region) of each electrode..$^{15}$ In these edge regions we expect that similar electrochemical conditions to the nanoband electrodes may well be able to be generated, which could result in a similarly nanostructured surface hydrogel with comparable edge sensing enhancement to these nanoelectrodes. In contrast, reflecting the markedly reduced mass transport, a more open surface hydrogel structure would be expected in the inner region. Given this inner region constitutes the vast majority of the electrode area and that the overall response of the microelectrode will be the combination of inner and edge region responses, the advantage of nanoband electrodes over microelectrodes is therefore likely to be the increased signal-to-noise, sensitivity and resistance to biofouling that results from the effective removal of this inner region and the exclusive production of a nanointerface.

\section{Availability of data}

Data used within this publication can be accessed at: http://dx.doi.org/10.7488/ds/ 2402.

\section{Conflicts of interest}

There are no conflicts to declare.

\section{Acknowledgements}

A. P. would like to thank the University of Edinburgh Schools of Chemistry and Engineering for funding his PhD studentship. D. J. A. thanks the EPSRC for 
a Fellowship (EP/L021978/1). The authors would like to acknowledge Dr A. Bunting and Prof. A. Walton from the Scottish Microelectronics Centre for their advice regarding the fabrication of the MNEE devices.

\section{References}

1 G. Liu, C. Sun, D. Li, S. Song, B. Mao, C. Fan and Z. Tian, Gating of redox currents at gold nanoelectrodes via DNA hybridization, Adv. Mater., 2010, 22(19), 2148-2150.

2 M. Silvestrini, L. Fruk and P. Ugo, Functionalized ensembles of nanoelectrodes as affinity biosensors for DNA hybridization detection, Biosens. Bioelectron., 2013, 40(1), 265-270.

3 A. Guiseppi-Elie, Electroconductive hydrogels: Synthesis, characterization and biomedical applications, Biomaterials, 2010, 31(10), 2701-2716.

4 W. Yang, H. Xue, L. R. Carr, J. Wang and S. Jiang, Zwitterionic poly(carboxybetaine) hydrogels for glucose biosensors in complex media, Biosens. Bioelectron., 2011, 26(5), 2454-2459.

5 M. V. Pishko, A. C. Michael and A. Heller, Amperometric glucose microelectrodes prepared through immobilization of glucose-oxidase in redox hydrogels, Anal. Chem., 1991, 63(20), 2268-2272.

6 J. A. Rowley, G. Madlambayan and D. J. Mooney, Alginate hydrogels as synthetic extracellular matrix materials, Biomaterials, 1999, 20(1), 45-53.

7 M. M. Dominguez, M. Wathier, M. W. Grinstaff and S. E. Schaus, Immobilized hydrogels for screening of molecular interactions, Anal. Chem., 2007, 79(3), 1064-1066.

8 K. Sirkar and M. V. Pishko, Amperometric biosensors based on oxidoreductases immobilized in photopolymerized poly(ethylene glycol) redox polymer hydrogels, Anal. Chem., 1998, 70(14), 2888-2894.

9 S. E. Salamifar and R. Y. Lai, Fabrication of electrochemical DNA sensors on gold-modified recessed platinum nanoelectrodes, Anal. Chem., 2014, 86(6), 2849-2852.

10 M. Wallace, D. J. Adams and J. A. Iggo, Analysis of the mesh size in a supramolecular hydrogel by PFG-NMR spectroscopy, Soft Matter, 2013, 9(22), 5483-5491.

$11 \mathrm{~N}$. Wisniewski and M. Reichert, Methods for reducing biosensor membrane biofouling, Colloids Surf., B, 2000, 18(3-4), 197-219.

12 J. G. Terry, I. Schmueser, I. Underwood, D. K. Corrigan, N. J. Freeman, A. S. Bunting, A. R. Mount and A. J. Walton, Nanoscale electrode arrays produced with microscale lithographic techniques for use in biomedical sensing applications, IET Nanobiotechnol., 2013, 7(4), 125-134.

13 I. Schmueser, A. J. Walton, J. G. Terry, H. L. Woodvine, N. J. Freeman and A. R. Mount, A systematic study of the influence of nanoelectrode dimensions on electrode performance and the implications for electroanalysis and sensing, Faraday Discuss., 2013, 164, 295-314.

14 Y. H. Lanyon, G. De Marzi, Y. E. Watson, A. J. Quinn, J. P. Gleeson, G. Redmond and D. W. M. Arrigan, Fabrication of nanopore array electrodes by focused ion beam milling, Anal. Chem., 2007, 79(8), 3048-3055. 
15 H. L. Woodvine, J. G. Terry, A. J. Walton and A. R. Mount, The development and characterisation of square microfabricated electrode systems, Analyst, 2010, 135(5), 1058-1065.

16 J. Raeburn, B. Alston, J. Kroeger, T. O. McDonald, J. R. Howse, P. J. Cameron and D. J. Adams, Electrochemically-triggered spatially and temporally resolved multi-component gels, Mater. Horiz., 2014, 1(2), 241-246; P. S. Kubiak, S. Awhida, C. Hotchen, W. Deng, B. Alston, T. O. McDonald, D. J. Adams and P. J. Cameron, Polymerization of low molecular weight hydrogelators to form electrochromic polymers, Chem. Commun., 2015, 51, 10427-10430.

17 A. Michnik, K. Michalik and Z. Drzazga, Stability of bovine serum albumin at different pH, J. Therm. Anal. Calorim., 2005, 80(2), 399-406.

18 X. Ji, C. E. Banks, D. S. Silvester, A. J. Wain and R. G. Compton, Electrode kinetic studies of the hydroquinone-benzoquinone system and the reaction between hydroquinone and ammonia in propylene carbonate: Application to the indirect electroanalytical sensing of ammonia, J. Phys. Chem. C, 2007, 111(3), 1496-1504.

19 W. J. Albery and A. R. Mount, Ring-disc electrodes 22. theory of the measurement of proton fluxes at the disk, J. Chem. Soc., Faraday Trans. 1, 1989, 85, 1181-1188.

20 W. J. Albery and A. R. Mount, Ring-disc electrodes 23. studies of proton fluxes at a thionine-coated electrode, J. Chem. Soc., Faraday Trans. 1, 1989, 85, 11891198.

21 A. J. Bard and L. R. Faulkner, Electrochemical Methods: Fundamentals and Applications, Wiley, New York, Chichester, 1980, p xviii, p. 718.

22 K. Ngamchuea, S. Eloul, K. Tschulik and R. G. Compton, Planar diffusion to macro disc electrodes-what electrode size is required for the Cottrell and Randles-Sevcik equations to apply quantitatively?, J. Solid State Electrochem., 2014, 18(12), 3251-3257.

23 N. Wisniewski, F. Moussy and W. M. Reichert, Characterization of implantable biosensor membrane biofouling, Fresenius'J. Anal. Chem., 2000, 366(6-7), 611621.

24 B. Yu, C. Wang, Y. M. Ju, L. West, J. Harmon, Y. Moussy and F. Moussy, Use of hydrogel coating to improve the performance of implanted glucose sensors, Biosens. Bioelectron., 2008, 23(8), 1278-1284.

25 D. K. Corrigan, H. Schulze, G. Henihan, A. Hardie, I. Ciani, G. Giraud, J. G. Terry, A. J. Walton, R. Pethig, P. Ghazal, J. Crain, C. J. Campbell, K. E. Templeton, A. R. Mount and T. T. Bachmann, Development of a PCRfree electrochemical point of care test for clinical detection of methicillin resistant Staphylococcus aureus (MRSA), Analyst, 2013, 138(22), 6997-7005.

26 C. M. A. Brett, S. Kresak, T. Hianik and A. M. O. Brett, Studies on selfassembled alkanethiol monolayers formed at applied potential on polycrystalline gold electrodes, Electroanalysis, 2003, 15(5-6), 557-565.

27 P. M. Kosaka, S. Gonzalez, C. M. Dominguez, A. Cebollada, A. San Paulo, M. Calleja and J. Tamayo, Atomic force microscopy reveals two phases in single stranded DNA self-assembled monolayers, Nanoscale, 2013, 5(16), 7425-7432. 
28 Z. Novakova, R. Orinakova, A. S. Fedorkova and A. Orinak, Electrochemical study of self-assembled monolayer adsorption, J. Solid State Electrochem., 2014, 18(8), 2289-2295.

29 T. Goda, E. Yamada, Y. Katayama, M. Tabata, A. Matsumoto and Y. Miyahara, Potentiometric responses of ion-selective microelectrode with bovine serum albumin adsorption, Biosens. Bioelectron., 2016, 77, 208-214.

30 E. M. Boon, J. E. Salas and J. K. Barton, An electrical probe of protein-DNA interactions on DNA-modified surfaces, Nat. Biotechnol., 2002, 20(3), 282-286.

31 K. Hirayama, S. Akashi, M. Furuya and K. Fukuhara, Rapid confirmation and revision of the primary structure of bovine serum-albumin by ESIMS and FRITFAB LC MS, Biochem. Biophys. Res. Commun., 1990, 173(2), 639-646. 